\title{
Vibratory gear adaptive transmission
}

\author{
Konstantin Ivanov ${ }^{1,2, ~ *}$, Zhilisbaeva K. S. ${ }^{3}$, Koilibaeva R. K. ${ }^{1,2}$ \\ ${ }^{1}$ Chair "Control Systems for Aerospace Engineering “, Almaty University of Power Engineering and Telecommunications, Almaty, \\ Kazakhstan \\ ${ }^{2}$ Laboratory of Adaptive Mechanisms, Institute of Mechanics and Machine Science MON RK, Almaty, Kazakhstan \\ ${ }^{3}$ Chair of Applied Mechanics, Kazakh National University named Al-Farabi, Almaty, Kazakhstan
}

Email address:

ivanovgreek@mail.ru (K. Ivanov)

To cite this article:

Konstantin Ivanov, Zhilisbaeva K. S., Koilibaeva R. K.. Vibratory Gear Adaptive Transmission. American Journal of Mechanics and Applications. Special Issue: Adaptive Transmissions. Vol. 2, No. 6-1, 2014, pp. 25-28. doi: 10.11648/j.ajma.s.2014020601.15

\begin{abstract}
Drives of machines which are used now do not possess ability to be adapted for extreme working conditions. Such conditions are connected with possible difficulties of motion because of deviations from operation norms (for example, because of long inactivity, deterioration of conditions of lubricant, minor damages, a temperature difference, etc.). In the conditions of impossibility of elimination of minor failures on the move (for example in aeronautical engineering) insignificant discrepancy of operation of service mechanism can serve as a cause of accident. Recently the technological direction of use of adaptive drive of machines is advanced. The adaptive drive mechanism contains the engine and the self-regulated transmission mechanism. The adaptive gear transmission mechanism with two degrees of freedom has ability to actuate an executive end-effector with a speed inversely to external load at constant engine power. It means that even in presence of handicap in motion of end-effector the drive mechanism breakage will not occur. The adaptive vibratory mechanism in the form of the closed gear differential with unbalanced masses can procure effective vibratory action on the end-effector. Such vibratory mechanism will procure the reliable overcoming of operational overloads. In offered work the describing of the vibratory mechanism with unbalanced masses is considered. Work is executed on the basis of mechanics laws.
\end{abstract}

Keywords: Gear Adaptive, Vibratory Acting, Differential Mechanism, Force Adaptation

\section{Introduction}

Vibratory gear adaptive transmission procures vibratory acting on output end-effector and adaptation of motion to variable force loading. Vibratory acting in a combination with force adaptation procures the possibility of reliable overcoming of starting resistance in the beginning of motion and overloads in an operational mode of motion of the machine.

In work of Ivanov [1, 2 and 3] the theoretical laws of creation of vibratory acting in an operational mode of motion when the kinematic chain is in a condition with two degrees of freedom are brought. As it is known the kinematic chain with two degrees of freedom having only one input is not definable [4]. However the presented laws of vibratory acting are based on the new theorem about the closed contour. This theorem contains the proof that the closed contour imposes constraint on relative motion of links and procures definability of motion of a kinematic chain. Thus the kinematic chain gets property of adaptation to variable technological loading.
The considered kinematic chain at start-up has a condition with one degree of freedom because the output link (the output carrier) is motionless during this moment. At start-up from a place the considered kinematic chain also will have uncertainty of motion because there is no resistance to motion on a link which precedes the motionless output carrier (that is on the satellite) on existing representations of the theory of mechanisms and machines in this chain.

Independent transition of a kinematic chain from a condition with one degree of freedom in a condition with two degrees of freedom becomes problematic without compulsory force acting on the satellite or without use of forces of a friction or inertial resistance.

Ivanov's last researches have shown that the closed four link contour imposes some constraint also on motion of a kinematic chain with one degree of freedom. It leads to definability of the kinematic chain motion.

In present article the proof of definability of motion 
vibratory gear stepless adaptive transfer is resulted at start-up and laws of vibratory acting on output end-effector are resulted at start-up in a condition with one degree of freedom.

Drives of machines which are using now do not possess ability to be accommodated or extreme working conditions. Such conditions are connected with possible difficulties of motion because of deviations from norms of maintenance (for example, because of long inactivity, a decline of conditions of greasing, insignificant faults, difference of temperatures, etc.). In the conditions of impossibility of elimination of small derangements on the move (for example, in an aeronautics) insignificant inaccuracy of maintenance of the service mechanism can serve as a cause of accident. The main deficiency of existing drives is a "rigid" constraint of the engine the executive tool through the connecting gear with one degree of freedom. In his case the insignificant handicap on the move the tool or its wedging can call breakage of the drive. Recently the scientific and technical direction of use of adaptive drives of machines develops [1-3]. The adaptive drive contains the engine and the self-controlled connecting gear. The adaptive gear connecting mechanism with two degrees of freedom has ability to set in motion the executive tool with speed which is inverse-proportional to external loading at constant power of engine. It means that drive breakage will not occur even in the presence of a handicap on the tool motion. he engine can transfer motion to the connecting gear even at tool dead stop. Conditions of start-up of such mechanism admit substantial growth of a starting force at the expense of small starting speed of tool at constant power of engine. It is offered to use conditions of vibratory affecting on the tool to procure the beginning of motion of the tool in the presence of a handicap. For this purpose it is offered to include elastic links into the drive mechanism. In the present work synthesis of vibratory transfer on the set maximum vibratory effort is presented.

Work is executed on the basis of mechanics laws.

\section{Description of Gear Adaptive Vibratory Mechanism}

The working out adaptive vibratory gear variator which procures a variable speed motion of the output tool depending on loading on him and creates vibratory affecting on the tool is considered. Vibratory affecting in a combination with force adaptation will procure reliable overcoming of starting resistance and overloading in operating conditions. Adaptive vibratory drive is shown on Fig. 1.

The mechanism contains frame 0 , input carrier $H_{1}$, input satellite 2, block of central toothed wheels with external teeth (solar wheels) $1-4$, block of central toothed wheels with internal teeth (ring wheels) $3-6$, output satellite 5 and output carrier $H_{2}$. Toothed wheels 4-1, 2, 3-6, 5 form the closed contour.

The external active moment $M_{H 1}$ is acting on the entrance carrier $H_{1}$. External moment of resistance $M_{H 2}$ is acting on the output carrier $\mathrm{H}_{2}$.

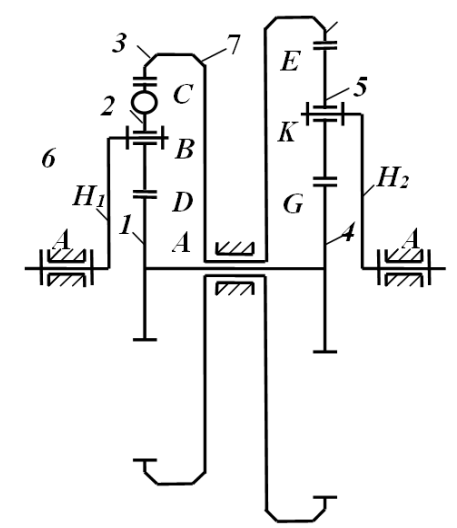

Figure 1. Gear adaptive vibratory mechanism

The output carrier $\mathrm{H}_{2}$ is moving quite definitely with a speed inversely proportional to output moment of resistance thanks to presence of two degree of freedom and differential constraint in the closed contour $[1,2]$ :

$$
\omega_{H 2}=M_{H 1} \omega_{H 1} / M_{H 2}
$$

Equation (1) expresses effect of force adaptation of output link to a variable load.

When the shafts connecting wheels $1-4$ and 3-6 are rigid angular speeds of wheels are defined under formulas [1,2 and 3]:

$$
\begin{gathered}
\omega_{3}=\frac{\omega_{H 2}\left(1-u_{46}^{(H 2)}\right)-\omega_{H 1}\left(1-u_{13}^{(H 1)}\right)}{u_{13}^{(H 1)}-u_{46}^{(H 2)}} \\
\omega_{1}=u_{46}^{(H 2)}\left(\omega_{3}-\omega_{H 2}\right)+\omega_{H 2}
\end{gathered}
$$

Here transfer ratios are next: $u_{13}^{(H 1)}=-z_{3} / z_{1}$, $u_{46}^{(H 2)}=-z_{6} / z_{4} \cdot u_{46}^{(H 2)}=-z_{6} / z_{4}$.

\section{Dynamic Analysis of Motion of Mechanical System with Elastic Shafts and Unbalanced Masse}

Presence of unbalanced masse $m$ and elastic shafts connecting wheels 1, 4 and 3,6 will lead to occurrence of dynamic pulses at transfer of motion on the output carrier.

Input carrier $H_{1}$ with the unbalanced satellite 2 is shown on Fig. 2.

Carrier $H_{1}$ transfers input motive force $F_{H 1}$ on satellite 2 . Force $F_{H 1}$ is transferred in points $C$ and $D$ to wheels 3 and 1 in the form of constantly operating forces $F_{H 1} / 2$.

Unbalanced masse $m$ creates on the satellite 2 centrifugal force of inertia

$$
I=m r \omega_{2}^{2}
$$

where $r$ - radius of an arrangement of the centre of masse $m$ on satellite $2, \omega_{2}$ - angular speed of satellite 2 .

Let's transfer force $I$ in the line of its action to point $B$ and we will spread out it to components. We will designate $\alpha$ angle of turn of radius $r$.

Component of inertia force $N=m r \omega_{2}^{2} \cos \alpha$ acts on frame. 
Component of inertia force $T=m r \omega_{2}^{2} \sin \alpha$ is transferred in points $C$ and $D$ to wheels 3 and 1 in the form of variable disturbing forces $T / 2$.

Constant motive forces $F_{H 1} / 2$ are transferred through blocks of wheels 1-4 and 3-6 on the output satellite 5 and further on the output carrier $\mathrm{H}_{2}$. These forces are overcoming the resistance force $R_{H_{2}}$ in point $K$. Variable disturbing forces $T / 2$ are overcoming elastic resistance forces in blocks of wheels 1-4 and 3-6 (as the wheels in each block are connected by elastic shaft). Disturbing forces create oscillations on wheels 4 and 6 which are transferred to the output satellite 5 and further on the output carrier $\mathrm{H}_{2}$ in the form of vibrations. These forces are creating the vibratory action in point $K$ which helps to overcome resistance force $R_{H 2}$ at overloads.

At the greatest possible loading the output carrier will stop and the mechanism will continue movement with one degree of freedom. If thus on one of wheels of the closed contour to place a flywheel with mobile masses fixed by means of springs [5]. This flywheel will create a stock of potential energy of springs. Preservation of a stock of potential energy of a flywheel provides the constant input driving moment on input carrier. On the output satellite 5 the moment of resistance corresponding to the driving moment will take place. Dynamic impulses from wheels $1-4$ and 3-6 will be transferred to the output satellite 5 and further on the motionless output carrier $H_{2}$. It will lead to resistance overcoming on motionless carrier both at emergency jamming and at start-up.

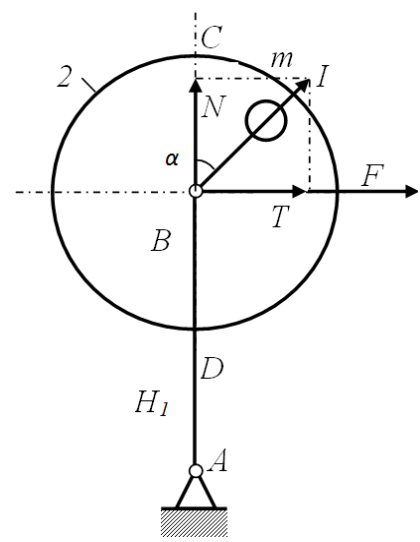

Figure 2. Input carrier with unbalanced satellite

Let's consider the possibility to receive of definability of system with two degrees of freedom when elastic shafts connect wheels 1 and 4 and wheels 3 and 6 .

Reactions $R_{23}=F_{H 1} / 2, R_{21}=F_{H 1} / 2$ are transferred on intermediate links 3 and 1 from entrance satellite 2. Reactions $R_{54}=R_{H 2} / 2, R_{56}=R_{H 2} / 2$ are transferred on intermediate links 4 and 6 from output satellite 5 .

Here $F_{H 1}=\frac{M_{H 1}}{r_{H 1}}, R_{H 2}=\frac{M_{H 2}}{r_{H 2}}, M_{H 1}, M_{H 2}$ - moments on input and output carriers, $r_{H 1}, r_{H 2}$ - radiuses of input and output carriers, $\quad r_{i}(i=1,2 \ldots 6)$ - radiuses of wheels.

Moments are transferred to wheels 4 and 6:

$$
\begin{aligned}
& M_{4}=0.5 M_{H 2} \frac{r_{4}}{r_{H 2}} \cdot \\
& M_{6}=0.5 M_{H 2} \frac{r_{6}}{r_{H 2}} .
\end{aligned}
$$

When elastic constraint between wheels 1-4 and 3-6 differential equations of motion of wheels 4 and 6 look like

$$
\begin{gathered}
J_{4} \ddot{\varphi}_{4}=M_{4}-c_{4}\left(\varphi_{4}-\varphi_{1}\right), \\
J_{6} \ddot{\varphi}_{6}=M_{6}-c_{6}\left(\varphi_{6}-\varphi_{3}\right) .
\end{gathered}
$$

Here $J_{4}, J_{6}-$ moments of inertia of wheels 4 and 6 ,

$c_{4}, c_{6}$ - torsion rigidity of the shafts connecting wheels $1-4$ and 3-6.

According to [4] on a method torsion rigidities at enough high power of the engine it is possible to consider angular speed $\omega_{1}$ as a constant, then $\varphi_{1}=\omega_{1} t$. The angle $\varphi_{4}$ differs from $\varphi_{1}$ a little. Therefore it is convenient to take for generalized co-ordinate a difference $\varphi=\varphi_{4}-\varphi_{1}$ instead $\varphi_{4}$. Then the differential equation (5) becomes:

$$
J_{4} \ddot{\varphi}+c_{4} \varphi=M_{4} .
$$

The solution of (7) at zero entry conditions looks like:

$$
\varphi=\frac{M_{4}}{c_{4}}\left[1-\cos \left(k_{4} t\right)\right] .
$$

Here $k_{4}=\sqrt{\frac{c_{4}}{J_{4}}}$ - oscillation frequency of wheel 4 concerning wheel 1 owing to elastics of the shaft connecting wheels 1-4.

Motion of a wheel 4 can be considered as the motion consisting of the basic motion with constant angular speed $\omega_{1}$ and additional motion with speed $\dot{\varphi}$ having oscillatory character:

$$
\dot{\varphi}=A_{\omega 4} \sin \left(k_{4} t\right)
$$

Here amplitude of angular speed $A_{\omega 4}=\frac{M_{4}}{c_{4}} k_{4}=\frac{M_{4}}{\sqrt{c_{4} J_{4}}}$.

Thus angular speed of wheel 4 in the presence of elastic constraint is

$$
\omega_{4}=\omega_{1}+\frac{M_{4}}{\sqrt{c_{4} J_{4}}} \sin \left(k_{4} t\right) .
$$

Angular speed of a wheel 6 is defined analogously:

$$
\omega_{6}=\omega_{3}+\frac{M_{6}}{\sqrt{c_{6} J_{6}}} \sin \left(k_{6} t\right) .
$$

Here $k_{6}=\sqrt{\frac{c_{6}}{J_{6}}}$ - oscillation frequency of wheel 6 concerning wheel 3 owing to elastics of the shaft connecting wheels 3 and 6 .

Thus for transfer of oscillations on the satellite 5 and the target carrier $\mathrm{H}_{2}$ without distortions (with frequency and 
amplitude of oscillation conservation) it is necessary to satisfy condition $k_{4}=k_{6}$. From this condition which is condition for the selection of rigid and inertia parameters follows:

$$
\frac{c_{6}}{c_{4}}=\frac{J_{6}}{J_{4}} \text {. }
$$

The calculations have shown that at performance of equation (13) the equality of amplitudes of oscillation of wheels 4 and 6 takes place also.

Amplitudes and periods of oscillations are selected by the set of shafts torsion rigidities and moments of inertia.

On the found angular speeds of wheels 4 and 6 the angular speed of target carrier $\mathrm{H}_{2}$ is defined:

$$
\omega_{H 2}=\frac{\omega_{4}-u_{46}^{H 2} \omega_{6}}{1-u_{46}^{H 2}}
$$

As is shown on Fig. 3 the angular speed $\omega_{H_{2}}$ of the target carrier $\mathrm{H}_{2}$ varies under the harmonious law about a mean $\omega_{H 20}$ defined by the Eq. 1 .

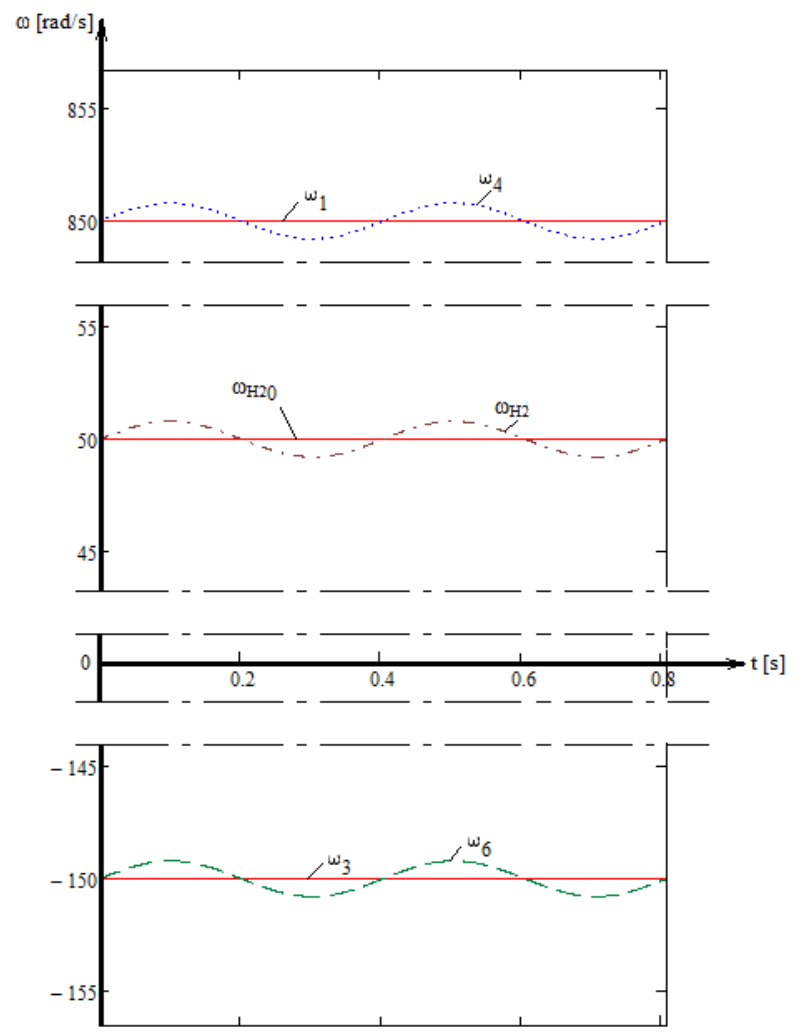

Figure 3. Diagram of change of angular speeds

\section{Synthesis of Vibratory Adaptive Transfer}

Synthesis consists in definition of parameters of the mechanism providing demanded vibratory acting, on following conditions:

1) Overcoming of starting extreme resistance on the motionless output carrier, exceeding normal resistance in set number of times (10 times) in a starting mode of motion,

2) Overcoming of extreme resistance on the moving output carrier exceeding normal resistance in set number of times (10 times) in an operational mode of motion.

The decision of a problem of synthesis of vibratory adaptive transfer is reduced to definition of parameters of a flywheel with mobile masses fixed by means of springs on a condition of overcoming of extreme resistance on the basis of equality potential and kinetic energy a flywheel with mobile masses fixed by means of springs.

\section{Conclusion}

The oscillation frequency in an elastic contour is high and has vibratory character. Vibratory affecting in combination with effect of force adaptation predetermines high reliability start and overcoming of emergency overloading.

The executed scientific researches allow creating simple and reliable adaptive vibratory drive of the service mechanism for the techniques working in extreme conditions (in aircraft, in space, etc).

The decision of a problem of synthesis of vibratory adaptive transfer is reduced to definition of parameters of a flywheel with mobile masses fixed by means of springs on a condition of overcoming of extreme resistance on the basis of equality potential and kinetic energy a flywheel with mobile masses fixed by means of springs.

\section{References}

[1] Ivanov K.S., Tultaev B. Toothed continuously variable transmission $(\mathrm{CVT})$ - industrial realization. New Trends in Mechanism and Machine Science. Theory and Applications in Engineering. V. 7. Springer. ISSN 2211-0984. Transactions of 4-th Euro Conference on Mechanisms. Springer. Santander. Spain. 329-335. 2012.

[2] Ivanov K.S., Koilybayeva R.K., Ualiev G.U. Creation of Vibratory Gear Continuously Variable Transmission (CVT). 11th International Conference on Vibratory Problems (ICOVP 2013). Book of Abstracts. Lisbon. Portugal. 2013. P 91.

[3] Ivanov K.S. Paradox of mechanics - a basis of creation CVT, Transactions of 2-d IFToMM Asian Conference on Mechanisms and Machines Science, Tokyo, Japan. P. 245 - 264. 2012.

[4] Levitsky N.I. Theory of mechanisms and machines. Moskow: Science, Main edition of physical and mathematical literature, 576 p. 1979.

[5] Ivanov K.S. Flywheel with variable distribution of masses. The copyright certificate. The patent of the USSR. №365503, 8th Jan 1973. 